\title{
Osciladores quânticos com massa dependente da posição
}

\section{Quantum oscillators with position-dependent mass}

\author{
J. P. G. Nascimento*1, I. Guedes ${ }^{1,2}$ \\ ${ }^{1}$ Departamento de Física, Universidade Federal do Ceará, Fortaleza, CE, Brasil \\ ${ }^{2}$ Seara da Ciência, Universidade Federal do Ceará, Fortaleza, CE, Brasil.
}

Recebido em 3 de dezembro de 2015. Revisado em 26 de janeiro de 2016. Aceito em 14 de fevereiro de 2016

Neste trabalho investigamos as soluções da equação de Schrödinger de osciladores com massa dependente da posição utilizando o método algébrico de operadores escada e uma transformação canônica de ponto. Os resultados são ilustrados para um sistema onde $m(x) \propto x^{2}$. De posse das funções de onda, calculamos a informação de Fisher e a entropia de Shannon para a posição e momentum de uma partícula no estado fundamental deste sistema.

Palavras-chave: Informação de Fisher, entropia de Shannon, massa dependente da posição, transformação canônica de ponto.

In this work we investigate the solutions of the Schrödinger equation of position-dependent mass oscillators by employing the algebraic method of ladder operators and a point canonical transformation. The results are illustrated for a system where $m(x) \propto x^{2}$. From the wave functions, we calculate the Fisher information and Shannon entropy in the position and momentum for a particle in the ground state of this system.

Keywords: Fisher information, Shannon entropy, position-dependent mass, point canonical transformation.

\section{Introdução}

Sistemas onde a massa depende da posição são importantes não apenas do ponto de vista teórico, mas também do ponto de vista prático, pois podem ser utilizados para descrever heteroestruturas abruptas ou suaves [1], no estudo das propriedades eletrônicas de semicondutores [2], de cristais homogêneos [3], de pontos [4] e líquidos quânticos [5]. Em 2015, Macedo e Guedes [6] resolveram a equação de Schrödinger e calcularam a Informação de Fisher e a e Entropia de Shannon para três diferentes osciladores com massa dependente da posição (OMDP). As distribuições de massa consideradas na Ref. [6] foram: (i) $m(x)=$ $\frac{m_{0}}{\left[(\lambda x)^{2}+a^{2}\right]^{2}}$, (ii) $m(x)=m_{0}\left[1+\tanh ^{2}(\lambda x)\right]$ e (iii) $m(x)=m_{0}\left[1+(\lambda x)^{2}\right]$. A primeira distribuição de massa é similar à distribuição $m(x)=\operatorname{sech}^{2}(\lambda x)$, que é utilizada na geração de uma sequência infinita de estados ligados para o problema de uma partícula livre [7]. A segunda distribuição é análoga as que

*Endereço de correspondência: joaopedro@fisica.ufc.br são utilizadas no estudo de estados eletrônicos confinados em estruturas do tipo $A l_{y} G a_{1-y} A s$ [8]. Já a terceira distribuição pode ser utilizada na análise das estruturas $G a A s / A l_{x} G a_{1-x} A s$ [1]

Recentemente, na Ref. [9], estudamos classicamente um OMDP cuja distribuição de massa era dada por $m(x)=m_{0}\left(x^{2}+\lambda^{2}\right)$, que é muito parecida com a distribuição de massa (iii) considerada por Macedo e Guedes [6]. Por meio do método da fatoração, estabelecemos uma correspondência entre as soluções do OMDP e do oscilador com massa constante (OMC). Analisamos ainda o espaço de fase do sistema para $\lambda=0,0,25$ e 1,0. Observamos que para $\lambda=0$, as trajetórias no espaço de fase são deformadas próximo à origem devido ao fato que $m(x) \rightarrow 0$ quando $x \rightarrow 0$. Para $\lambda \neq 0$, a deformação diminui e praticamente desaparece para $\lambda=1,0$. Mostramos assim que para sistemas tipo OMDP para os quais as equações de Hamilton ficam muito complicadas, a análise das trajetórias no espaço de fase fornece uma boa descrição da dinâmica do sistema em consideração. 
O objetivo deste trabalho, como uma extensão natural daquele apresentado na Ref. [9], é obter as soluções da equação de Schrödinger para o sistema com $m(x)=m_{0}\left(x^{2}+\lambda^{2}\right)$, e de posse dessas soluções calcular a informação de Fisher e a entropia de Shannon para o estado fundamental.

Este trabalho encontra-se assim dividido. $\mathrm{Na}$ seção 2, mostraremos como obter a forma do operador energia cinética, $T(x)$, e resolver a correspondente equação de Schrödinger para sistemas OMDP usando o método algébrico de operadores escada e uma transformação canônica de ponto. Na Seção 3, calculamos a informação de Fisher e a entropia de Shannon para o sistema considerado e apresentamos uma análise dos resultados. Por fim, na Seção 4, apresentamos alguns comentários finais.

\section{Solução da equação de Schrödinger - Álgebra de Heisenberg e a TCP}

Na Ref. [9], vimos que para uma dada distribuição de massa $m(x)$, o hamiltoniano clássico é dado por

$$
H=T(x)+V(x)
$$

onde $T(x)=\frac{p^{2}}{2 m(x)}$ e $V(x)=\frac{1}{2}\left(\int \sqrt{m(y)} d y+X_{0}\right)^{2}$, com $X_{0}$ sendo uma constante de integração.

Infelizmente, a extensão do hamiltoniano clássico para o quântico em casos onde a massa depende da posição, não é trivial como em casos onde a massa é constante ou varia com o tempo. O problema surge do fato que para sistemas com massa dependente da posição, a massa não comuta com o momentum. De acordo com von Roos [10], para que o hamiltoniano que descreve o sistema seja hermitiano, o operador energia cinética deve ser escrito como

$$
T=\frac{1}{4}\left(m^{\alpha} p m^{\beta} p m^{\gamma}+m^{\gamma} p m^{\beta} p m^{\alpha}\right),
$$

onde $m=m(x), p=-i \hbar d / d x$ e $\alpha, \beta$ e $\gamma$ satisfazem a relação de vínculo

$$
\alpha+\beta+\gamma=-1
$$

Dessa forma, a equação de Schrödinger independente do tempo (ES) para estados estacionários é dada por

$$
H \psi(x)=E \psi(x),
$$

Com $T(x)$ sendo dado pela Eq. (2) no caso de OMDP.

Existem diversos artigos que discutem sobre qual é a forma correta do operador $T(x)$. Por exemplo, em 1966, BenDaniel e Duke [11] consideraram $\alpha=\gamma=$ $0, \beta=-1$. Em 1969, Gora e Williams [12] estudaram o caso $\alpha=-1, \beta=\gamma=0$. Zhu e Kroemer [13], em 1983, analisaram sistemas com $\alpha=\gamma=-\frac{1}{2}, \beta=0$ e, em 1993, o caso $\alpha=0, \beta=\gamma=-\frac{1}{2}$ foi estudado por Li e Kuhn [14].

Como mencionamos na Ref. [9], o procedimento utilizado para obter as soluções da equação de Schrödinger para sistemas tipo OMDP é baseado em buscar transformações que permitam tratar o problema de OMDP da mesma forma que fazemos para sistemas tipo OMC. Assim, inicialmente consideremos o OMC ( $\operatorname{com} m=1)$. O espectro de energia e os autoestados do hamiltoniano $h=-\frac{1}{2} \frac{d^{2}}{d y^{2}}+\frac{1}{2} y^{2}$, onde consideramos $\hbar=1$, podem ser obtidos de duas maneiras [15], a saber:

(a) Método algébrico, que consiste em fatorar o hamiltoniano em termos de dois operadores, $b^{-} \mathrm{e}$ $b^{+}$, definidos como

$$
b^{ \pm}=\frac{1}{\sqrt{2}}\left(\mp \frac{d}{d y}+y\right) .
$$

Neste caso o hamiltoniano é dado por

$$
h=-\frac{1}{2} \frac{d^{2}}{d y^{2}}+\frac{1}{2} y^{2}=b^{+} b^{-}+\frac{1}{2} .
$$

Pode-se mostrar facilmente que estes operadores satisfazem as seguintes propriedades:

(a.1) Os operadores $b^{-}$e $b^{+}$são conjugados hermitianos (auto-adjuntos)

$$
\left(b^{ \pm}\right)^{\dagger}=b^{\mp}
$$

(a.2) Os operadores $b^{-}$e $b^{+}$obedecem à álgebra de Heisenberg

$$
\begin{gathered}
{\left[b^{-}, b^{+}\right]=1,} \\
{\left[h, b^{ \pm}\right]= \pm b^{ \pm} .}
\end{gathered}
$$

Como consequência dessas propriedades, e considerando $\phi_{0}$ como o estado fundamental do sistema 
descrito por $h$ (veja Eq. (6)), temos as seguintes relações

$$
\begin{gathered}
b^{-} \varphi_{0}(y)=0, \\
b^{-} \varphi_{n}(y)=\sqrt{n} \varphi_{n-1}(y), \\
b^{+} \varphi_{n}(y)=\sqrt{n+1} \varphi_{n+1}(y), \\
\varphi_{n}(y)=\frac{1}{\sqrt{n !}}\left(b^{+}\right)^{n} \varphi_{0}(y), \\
E_{n}=n+\frac{1}{2} .
\end{gathered}
$$

Resolvendo a Eq. (9), obtemos o estado fundamental e, aplicando sucessivamente o operador $b^{+}$ sobre $\phi(y)$, chegamos aos demais autoestados (Eq. (12)).

(b) Método analítico, que consiste em resolver diretamente a equação diferencial associada à equação de Schrödinger. Levando-se em consideração a normalização de estados ligados, as autofunções normalizadas $\left(\phi_{n}(y)\right)$ são identificadas em termos dos polinômios de Hermite expressos por

$$
\varphi_{n}(y)=\frac{1}{\sqrt{\pi^{1 / 2} 2^{n} n !}} e^{-y^{2} / 2} H_{n}(y) .
$$

Voltemos agora para o problema da solução da equação de Schrödinger para o OMDP (veja Eq. (4)). Considerando $\alpha=\gamma=a$ e $\beta=2 b$, podemos reescrever o operador energia cinética como

$$
T=\frac{1}{2} m^{a}(x) p m^{2 b}(x) p m^{a}(x),
$$

com a nova relação de vínculo sendo dada por $a+b$ $=-1 / 2$. Uma vez que $H_{a}$ é dado por

$$
H_{a}=-\frac{1}{2} m^{a}(x) \frac{d}{d x} m^{2 b}(x) \frac{d}{d x} m^{a}(x)+V_{a}(x),
$$

vamos supor que possamos fatorá-lo na forma

$$
H_{a}=A_{a}^{+} A_{a}^{-}+\frac{1}{2},
$$

utilizando os seguintes operadores auto-adjuntos

$$
\begin{gathered}
A_{a}^{-}=\frac{1}{\sqrt{2}} m^{b}(x) \frac{d}{d x} m^{a}(x)+W_{a}(x), \\
A_{a}^{+}=-\frac{1}{\sqrt{2}} m^{a}(x) \frac{d}{d x} m^{b}(x)+W_{a}(x),
\end{gathered}
$$

onde $W_{a}(x)$ é denominado de superpotencial. Observe que podemos ainda reescrever as Eqs. (18) na forma

$$
\begin{aligned}
A_{a}^{-} & =\frac{1}{\sqrt{2 m(x)}}\left[\frac{d}{d x}+a[\ln m(x)]^{\prime}\right] \\
& +W_{a}(x)
\end{aligned}
$$

$$
\begin{aligned}
A_{a}^{+} & =-\frac{1}{\sqrt{2 m(x)}}\left[\frac{d}{d x}+b[\ln m(x)]^{\prime}\right] \\
& +W_{a}(x)
\end{aligned}
$$

onde' significa derivada com respeito a $x$. A relação entre $V_{a}(x)$ e $W_{a}(x)$ é obtida da seguinte maneira. Considerando uma função arbitrária $f(x)$, obtemos a partir da Eq. (16) que

$$
\begin{aligned}
H_{a} f & =\left[-\frac{1}{2} m^{a} \frac{d}{d x} m^{2 b} \frac{d}{d x} m^{a}+V_{a}(x)\right] f(x) \\
& =-\frac{1}{2}\left\{m^{-1} f^{\prime \prime}-m^{-2} m^{\prime} f^{\prime}+a\left[m^{-2} m^{\prime \prime}\right.\right. \\
& \left.\left.-(a+2)\left(m^{\prime}\right)^{2} m^{-3}\right] f\right\}+V_{a} f .
\end{aligned}
$$

Por outro lado, utilizando a Eq. (17) combinada com as Eqs. (19.a) e (19.b) temos

$$
\begin{aligned}
H_{a} f & =\left[A_{a}^{+} A_{a}^{-}+\frac{1}{2}\right] f(x)=-\frac{1}{2} m^{-1} f^{\prime \prime} \\
& +\frac{1}{2} m^{-2} m^{\prime} f^{\prime}+f\left\{\frac{1}{2} a^{2} m^{-3}\left(m^{\prime}\right)^{2}\right. \\
& \left.+\frac{1}{\sqrt{2}}\left(\frac{4 a+1}{2}\right) m^{-\frac{3}{2}} m^{\prime} W_{a}\right\} \\
& +f\left\{-\frac{1}{2} a m^{-2} m^{\prime \prime}-\frac{1}{\sqrt{2}} W_{a}^{\prime} m^{-\frac{1}{2}}\right. \\
& \left.+W_{a}^{2}+a m^{-3}\left(m^{\prime}\right)^{2}+\frac{1}{2}\right\} .
\end{aligned}
$$


Comparando as Eqs. (20) e (21), obtemos a seguinte expressão

$$
\begin{aligned}
V_{a}(x) & =\frac{1}{\sqrt{2 m(x)}}\left[\frac{(4 a+1)}{2} W_{a}(x)\right. \\
& \left.\times[\ln m(x)]^{\prime}-W_{a}^{\prime}(x)\right]+W_{a}^{2}(x)+\frac{1}{2} .
\end{aligned}
$$

Não é difícil mostrar que a relação de comutação entre $A_{a}^{-}$e $A_{a}^{+}$é dada por

$$
\begin{array}{r}
{\left[A_{a}^{-}, A_{a}^{+}\right]=\sqrt{\frac{2}{m(x)}} W_{a}^{\prime}(x)+\left(\frac{4 a+1}{4}\right)} \\
\times \frac{1}{\sqrt{m(x)}} \frac{d}{d x}\left\{\frac{1}{\sqrt{m(x)}}[\ln m(x)]^{\prime}\right\} .
\end{array}
$$

Impondo que $\left[A_{a}^{-}, A_{a}^{+}\right]=1$, obtemos que $W_{a}(x)$ é expresso por

$$
\begin{aligned}
& W_{a}(x)=\frac{1}{\sqrt{2}}\left\{\int^{x} \sqrt{m(y)} d y+X_{0}\right. \\
& \left.-\left(\frac{4 a+1}{4}\right) \frac{1}{\sqrt{m(x)}}[\ln m(x)]^{\prime}\right\} .
\end{aligned}
$$

Substituindo a Eq. (24) na Eq. (22) chegamos à seguinte expressão para $V_{a}(x)$

$$
\begin{aligned}
& V_{a}(x)=\frac{1}{2}\left(\int \sqrt[x]{m(y)} d y+X_{0}\right)^{2} \\
& +\frac{1}{2}\left(\frac{4 a+1}{4}\right) \frac{1}{\sqrt{m(x)}} \frac{d}{d x}\left\{\frac{1}{\sqrt{m(x)}}[\ln m(x)]^{\prime}\right\} \\
& -\frac{1}{2}\left[\left(\frac{4 a+1}{4}\right) \frac{1}{\sqrt{m(x)}}[\ln m(x)]^{\prime}\right]^{2} .
\end{aligned}
$$

Na Ref. [9] mostramos que a expressão clássica de $V_{a}(x)$ é dada por

$$
V_{a}(x)=\frac{1}{2}\left(\int^{x} \sqrt{m(y)} d y+X_{0}\right)^{2} .
$$

Assim, para que haja concordância entre as expressões clássica e quântica de $V_{a}(x)$, devemos escolher $a=-1 / 4$. Dessa forma, a hamiltoniana quântica do sistema é dada simplesmente por

$$
\begin{aligned}
& H_{-1 / 4}(x, p)=A_{-1 / 4}^{+} A_{-1 / 4}^{-}+\frac{1}{2} \\
& =-\frac{1}{2} \frac{1}{\sqrt[4]{m(x)}} \frac{d}{d x} \frac{1}{\sqrt{m(x)}} \frac{d}{d x} \frac{1}{\sqrt[4]{m(x)}} \\
& +\frac{1}{2}\left(\int \sqrt[x]{m(y)} d y+X_{0}\right)^{2}
\end{aligned}
$$

onde a constante $X_{0}$ é usualmente tomada de maneira a tornar $V_{a}(x=0)=0$.

De maneira análoga ao OMC quântico, as propriedades de $A_{-1 / 4}^{+}$e $A_{-1 / 4}^{-}$garantem que

$$
A_{-1 / 4}^{-} \psi_{0}(x)=0
$$

$$
\begin{gathered}
A_{-1 / 4}^{-} \psi_{n}(x)=\sqrt{n} \psi_{n-1}(x), \\
A_{-1 / 4}^{+} \psi_{n}(x)=\sqrt{n+1} \psi_{n+1}(x),
\end{gathered}
$$

$$
\psi_{n}(x)=\frac{1}{\sqrt{n !}}\left(A_{-1 / 4}^{+}\right)^{n} \psi_{0}(x),
$$

com a equação de autovalores de $H_{-1 / 4}(x, p)$ sendo expressa por

$$
H_{-1 / 4}(x, p) \psi_{n}(x)=E_{n} \psi_{n}(x),
$$

onde $E_{n}=n+1 / 2$.

A TCP consiste em considerarmos as seguintes substituições na Eq. (32)

$$
\begin{gathered}
X=X(x)=\int \sqrt{m(y)} d y, \\
\psi_{n}(x)=\sqrt[4]{m(x)} \xi_{n}[X(x)],
\end{gathered}
$$

obtendo assim a expressão

$$
-\frac{1}{2} \frac{d^{2} \xi_{n}(X)}{d X^{2}}+\frac{1}{2}\left(X+X_{0}\right)^{2} \xi_{n}(X)=E_{n} \xi_{n}(X),
$$

ou seja, a equação de autovalores do OMC deslocado de $-X_{0}$, cujas soluções são bastante conhecidas. Logo, as autofunções de $H_{-1 / 4}(x, p)$ serão dadas por

$$
\begin{aligned}
\psi_{n}(x) & =\frac{1}{\sqrt{\pi^{1 / 2} 2^{n} n !}} \sqrt[4]{m(x)} \\
& \times \exp \left[-\frac{1}{2}\left(\int \sqrt{m(y)} d y+X_{0}\right)^{2}\right] \\
& \times H_{n}\left[\int \sqrt{m(y)} d y+X_{0}\right] .
\end{aligned}
$$


Estas soluções satisfazem automaticamente a seguinte condição de normalização

$$
\begin{aligned}
& \int\left|\psi_{n}(x)\right|^{2} d x=\int \sqrt{m(x)}\left|\xi_{n}(X)\right|^{2} \frac{d x}{d X} d X \\
& =\int \sqrt{m(x)}\left|\xi_{n}(X)\right|^{2} \frac{1}{\sqrt{m(x)}} d X \\
& =\int\left|\xi_{n}(X)\right|^{2} d X=1 .
\end{aligned}
$$

\section{Resultados e discussão - Princípio da Incerteza de Heisenberg, Informação de Fisher e Entropia de Shannon}

A partir das soluções da equação de Schrödinger dadas pela Eq.(36) podemos obter os valores de várias grandezas físicas, incluindo as incertezas na posição $(\Delta x)$ e no momentum $(\Delta p)$ que são utilizadas no cálculo do Princípio de Incerteza de Heisenberg (PIH) [16], que impõe à mecânica quântica um caráter intrínseco sobre incertezas nas medidas de observáveis físicos. O PIH nos diz que para um dado sistema físico a seguinte relação deve ser obedecida: $\Delta x \Delta p \geqslant \hbar / 2$. Qualitativamente, o PIH estabelece um limite inferior ao produto das incertezas da posição e do momento, revelando um aspecto da natureza, em nível atômico, totalmente diverso daquele a que estamos habituados, ou seja, quanto mais sabemos acerca da posição da partícula, menos podemos dizer a respeito de seu momento, e viceversa. Devido ao fato de as incertezas $(\Delta x)$ e $(\Delta p)$ serem obtidas a partir da densidade de probabilidade da função de onda $\left(\left|\psi_{n}(x)\right|^{2}\right)$, dizemos que o PIH é associado a medidas globais de $\left|\psi_{n}(x)\right|^{2}$.

Recentemente, foram propostas diferentes relações de incerteza, as chamadas relações de incerteza entrópicas, que podem fornecer novos limites inferiores ao produto das incertezas. Entre outras, mencionamos a relação de incerteza obtida a partir da informação de Fisher e da entropia de Shannon.

A informação de Fisher foi estabelecida em 1925 [17] como uma maneira de medir a quantidade de informação que um observável $X$ carrega em relação a um dado parâmetro $\theta$ para o qual a densidade de probabilidade de $X$ varia, ou seja, para o qual a densidade de probabilidade seja uma função explícita de $X$ e $\theta, \mathcal{P}(X, \theta)$

A entropia de Shannon [18] estabelece o conceito de informação e define-o como tudo aquilo que reduz a incerteza. Essa incerteza é definida para cada conjunto de elementos que se tenha. Por exemplo, como varia a idade dos alunos de uma sala. A Entropia de Shannon para um conjunto de elementos discretos $X=\left\{x_{i}\right\}$, onde a cada elemento está associada uma densidade de probabilidade $\left\{\mathcal{P}\left(x_{i}\right)\right\}$, é dada por

$$
S_{X}=-\sum_{i=1}^{n} \mathcal{P}\left(x_{i}\right) \ln \mathcal{P}\left(x_{i}\right) .
$$

Observe que se temos certeza do resultado de um dado experimento, ou seja, se o conjunto $X$ for constituído de um único elemento $x_{1}$, então $\mathcal{P}\left(x_{1}\right)=$ 1 e $\mathcal{P}\left(x_{i}\right)=0$ para $i \neq 1$. Dessa maneira, a entropia de Shannon está relacionada com o grau de incerteza e quantifica, portanto, a informação. Quanto menor for $S_{X}$ menor é o grau de incerteza e maior será a informação que se tem.

A informação de Fisher de um observável unidimensional $\theta(\in \mathbb{R})$ com uma densidade de probabilidade $\mathcal{P}(\theta)$ é dada por [19]

$$
F_{\theta}=\int \mathcal{P}(\theta)\left[\frac{d \ln \mathcal{P}(\theta)}{d \theta}\right]^{2} d \theta \geqslant 0 .
$$

Assim, a informação de Fisher da posição de um sistema quântico, cuja densidade de probabilidade no espaço das posições é $\mathcal{P}(x)=\left|\psi_{n}(x, t)\right|^{2}$, será

$$
\begin{aligned}
F_{x} & =\int\left|\psi_{n}(x, t)\right|^{2}\left[\frac{d \ln \left|\psi_{n}(x, t)\right|^{2}}{d x}\right]^{2} d x \\
& =\int \psi_{n}(x, t) \psi_{n}^{*}(x, t)\left[\frac{d \ln \left[\psi_{n}(x, t) \psi_{n}^{*}(x, t)\right]}{d x}\right]^{2} d x,
\end{aligned}
$$

ou seja,

$$
\begin{aligned}
F_{x} & =4 \int \psi_{n}^{*^{\prime}}(x, t) \psi_{n}^{\prime}(x, t) d x \\
& +\int\left[\frac{\psi_{n}^{\prime}(x, t)}{\psi_{n}(x, t)}-\frac{\psi_{n}^{* \prime}(x, t)}{\psi_{n}^{*}(x, t)}\right]^{2}\left|\psi_{n}(x, t)\right|^{2} d x .
\end{aligned}
$$

Da mesma maneira, a informação de Fisher para o momento será dada por

$$
\begin{aligned}
F_{p} & =4 \int \varphi_{n}^{*^{\prime}}(p, t) \varphi_{n}^{\prime}(p, t) d p \\
& +\int\left[\frac{\varphi_{n}(p, t)}{\varphi_{n}(p, t)}-\frac{\varphi_{n}^{*^{\prime}}(p, t)}{\varphi_{n}^{*}(p, t)}\right]^{2}\left|\varphi_{n}(p, t)\right|^{2} d p,
\end{aligned}
$$


onde $\varphi(p, t)$ é a transformada de Fourier de $\psi(x, t)$, ou seja

$$
\varphi(p, t)=\frac{1}{(2 \pi \hbar)^{1 / 2}} \int e^{-i p x / \hbar} \psi(x, t) d x .
$$

Como mostram as Eqs. (40) e (41), as informações de Fisher são associadas a medidas locais das densidades de probabilidades. A informação de Fisher e os desvios padrão de posição e momento de osciladores harmônicos estão relacionadas pelas inequações de Stam [20] e Cramer-Rao [21], dadas respectivamente, por

$$
F_{x} \leqslant 4(\Delta p)^{2}, F_{p} \leqslant 4(\Delta x)^{2},
$$

e

$$
F_{x} \geqslant \frac{1}{(\Delta x)^{2}}, F_{p} \geqslant \frac{1}{(\Delta p)^{2}} .
$$

Por sua vez, a entropia de Shannon de um observável unidimensional $\theta$ com uma densidade de probabilidade $\mathcal{P}(\theta)$ é dada por [22]

$$
S_{\theta}=-\int \mathcal{P}(\theta) \ln \mathcal{P}(\theta) d \theta
$$

Assim, as entropias de Shannon no espaço da posição e do momento são, respectivamente

$$
\begin{aligned}
& S_{x}=-\int\left|\psi_{n}(x, t)\right|^{2} \ln \left|\psi_{n}(x, t)\right|^{2} d x, \\
& S_{p}=-\int\left|\varphi_{n}(p, t)\right|^{2} \ln \left|\varphi_{n}(p, t)\right|^{2} d p,
\end{aligned}
$$

que também são associadas a medidas globais das densidades de probabilidades. A partir das expressões para $S_{x}$ e $S_{p}$, podemos estabelecer a seguinte relação de incerteza entrópica $[23,24]$ para problemas unidimensionais

$$
S=S_{x}+S_{p} \geqslant(1+\ln \pi) .
$$

Substituindo $m(x)=m_{0}\left(x^{2}+\lambda^{2}\right)$ na Eq. (36), os estados estacionários para este sistema são

$$
\begin{aligned}
\psi_{n}(x, t) & =\frac{1}{\sqrt{\pi^{1 / 2} 2^{n} n !}} \sqrt[4]{m_{0}\left(x^{2}+\lambda^{2}\right)} \exp \left(-i E_{n} t\right) \\
& \times \exp \left[-\frac{1}{8}\left\{x \sqrt{x^{2}+\lambda^{2}}+\lambda^{2} \ln \left[2\left(x+\sqrt{x^{2}+\lambda^{2}}\right)\right]-\lambda^{2} \ln (2 \lambda)\right\}^{2}\right] \\
& \times H_{n}\left[\frac{1}{2}\left(x \sqrt{x^{2}+\lambda^{2}}+\lambda^{2} \ln \left[2\left(x+\sqrt{x^{2}+\lambda^{2}}\right)\right]-\lambda^{2} \ln (2 \lambda)\right)\right]
\end{aligned}
$$

onde consideramos $m_{0}=1$ e $X_{0}=-\frac{\lambda^{2} \ln (2 \lambda)}{2}$.

Como o princípio de incerteza estabelecido para o OMC é mínimo para o estado fundamental, vamos considerar apenas a função de onda para $n=0$ no cálculo das informações de Fisher e entropias de Shannon. Neste caso, temos que $\psi_{0}(x, t)$ é dada por

$$
\psi_{0}(x, t)=\sqrt[4]{\frac{m_{0}\left(x^{2}+\lambda^{2}\right)}{\pi}} \exp \left(-i E_{0} t\right) \exp \left[-\frac{1}{8}\left\{x \sqrt{x^{2}+\lambda^{2}}+\lambda^{2} \ln \left[2\left(x+\sqrt{x^{2}+\lambda^{2}}\right)\right]-\lambda^{2} \ln (2 \lambda)\right\}^{2}\right]
$$

As Figs. 1(a) e 1(b) mostram as densidades de probabilidade no espaço da posição $\mathcal{P}_{0}(x)=\left|\psi_{0}(x)\right|^{2} \mathrm{e}$ do momento $\mathcal{P}_{0}(p)=\left|\varphi_{0}(p)\right|^{2}$ para $\lambda=0, \lambda=0,2$ e $\lambda=0,4$, enquanto as Figs. 2(a) e 2(b) mos- tram as mesmas quantidades, $\mathcal{P}_{0}(x)=\left|\psi_{0}(x)\right|^{2}$ e $\mathcal{P}_{0}(p)=\left|\varphi_{0}(p)\right|^{2}$, para $\lambda=0,6, \lambda=0,8$ e $\lambda=1$.

Nas Figs. 3(a) e 3(b) mostramos a variação das incertezas na posição $\left(\Delta x_{0}\right)$ e no momentum $\left(\Delta p_{0}\right)$ 

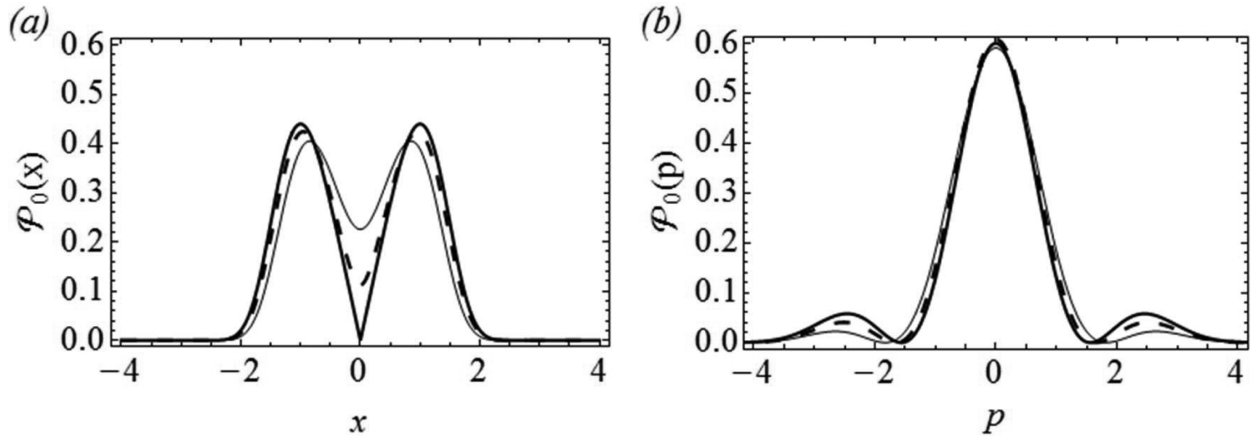

Figura 1: Densidades de probabilidade no espaço da posição $\mathcal{P}_{0}(x)=\left|\psi_{0}(x)\right|^{2}$ e do momento $\mathcal{P}_{0}(p)=\left|\varphi_{0}(p)\right|^{2}$ para $\lambda=0$ (linha grossa), $\lambda=0,2$ (linha pontilhada) e $\lambda=0,4$ (linha fina). Nos cálculos consideramos $m_{0}=1$.
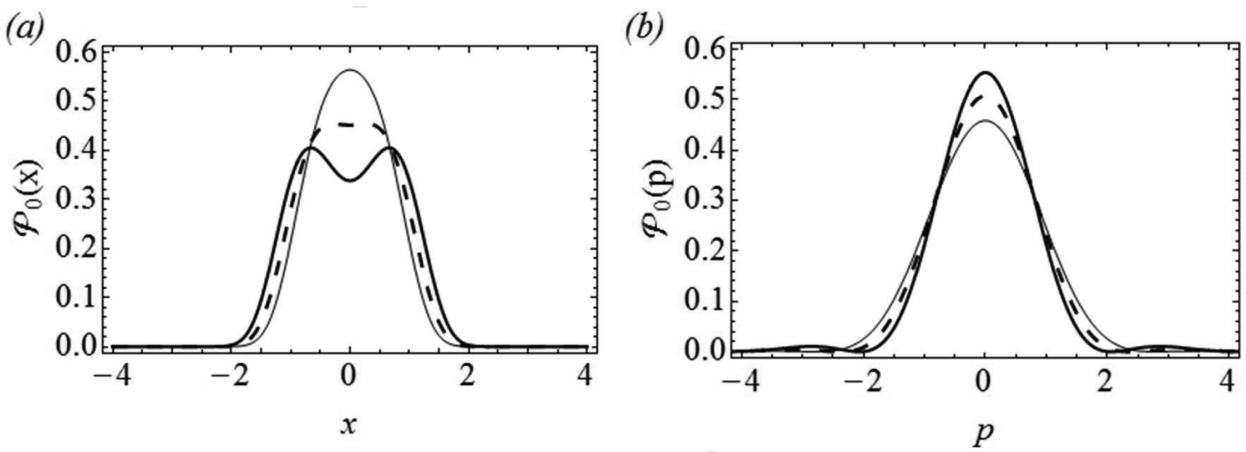

Figura 2: Densidades de probabilidade no espaço da posição $\mathcal{P}_{0}(x)=\left|\psi_{0}(x)\right|^{2}$ e do momento $\mathcal{P}_{0}(p)=\left|\varphi_{0}(p)\right|^{2}$ para $\lambda=0,6$ (linha grossa), $\lambda=0,8$ (linha pontilhada) e $\lambda=1$ (linha fina). Nos cálculos consideramos $m_{0}=1$.
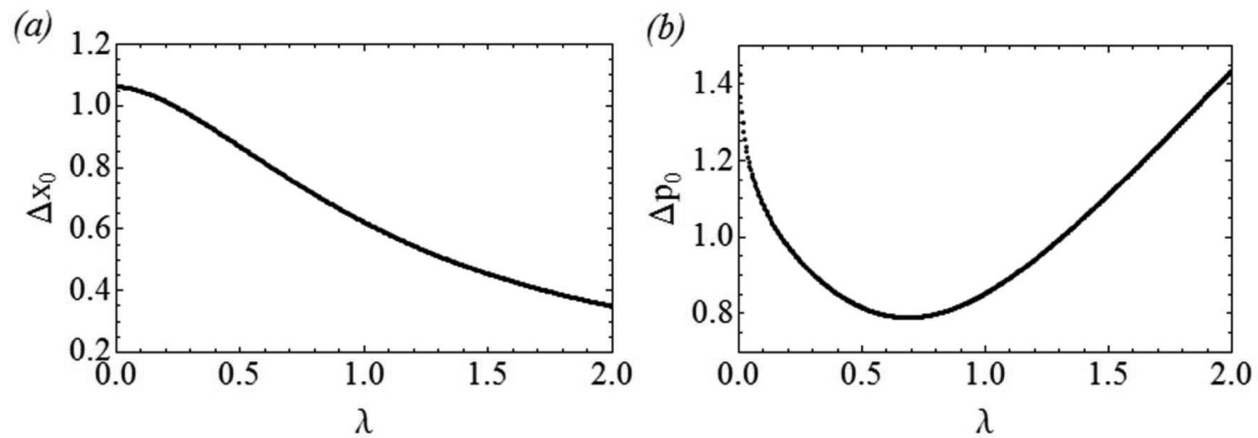

Figura 3: Dependência das incertezas (a) $\Delta x_{0}$ e (b) $\Delta p_{0}$ com relação ao parâmetro $\lambda$. Nos cálculos consideramos $m_{0}=1$.

em função do parâmetro $\lambda$. Ao aumentarmos $\lambda$, observamos que $\Delta x_{0}$ diminui. Podemos entender este resultado a partir da variação de $\mathcal{P}_{0}(x)$ mostrada nas Figs. 1(a) e 2(a). Observamos que a largura de $\mathcal{P}_{0}(x)$ diminui, o que torna a partícula mais localizada à medida em que $\lambda$ aumenta, diminuindo a incerteza na posição. Nesse cenário devíamos esperar que tanto a incerteza para o momento, $\Delta p_{0}$, como a informação de Fisher na posição, $F_{x}$, também aumentassem, enquanto a entropia de Shannon, $S_{x}$, diminuísse. Entretanto, observamos da Fig. 3(b) que à medida em que $\lambda$ aumenta, $\Delta p_{0}$ diminui no intervalo $0 \leqslant \lambda \leqslant 0,6$, ao invés de aumentar como esperado. Para $\lambda>0,6, \Delta p_{0}$ sempre aumenta.

Nas Figs. 4(a) e 4(b) mostramos o comportamento de $F_{x}$ e $F_{p}$, enquanto nas Figs. $5(\mathrm{a})$ e $5(\mathrm{~b})$ os de $S_{x}$ e $S_{p}$, em função da variação de $\lambda$. Consideremos as variações de $F_{x}$ e $F_{p}$. Como já mencionado, deveríamos esperar um aumento de $F_{x}$ e uma diminuição de $S_{x}$; o contrário ocorrendo para $F_{p}$ e $S_{p}$. Entretanto, observamos que no intervalo $0 \leqslant \lambda \leqslant 0,6$ tanto $F_{x}$ 

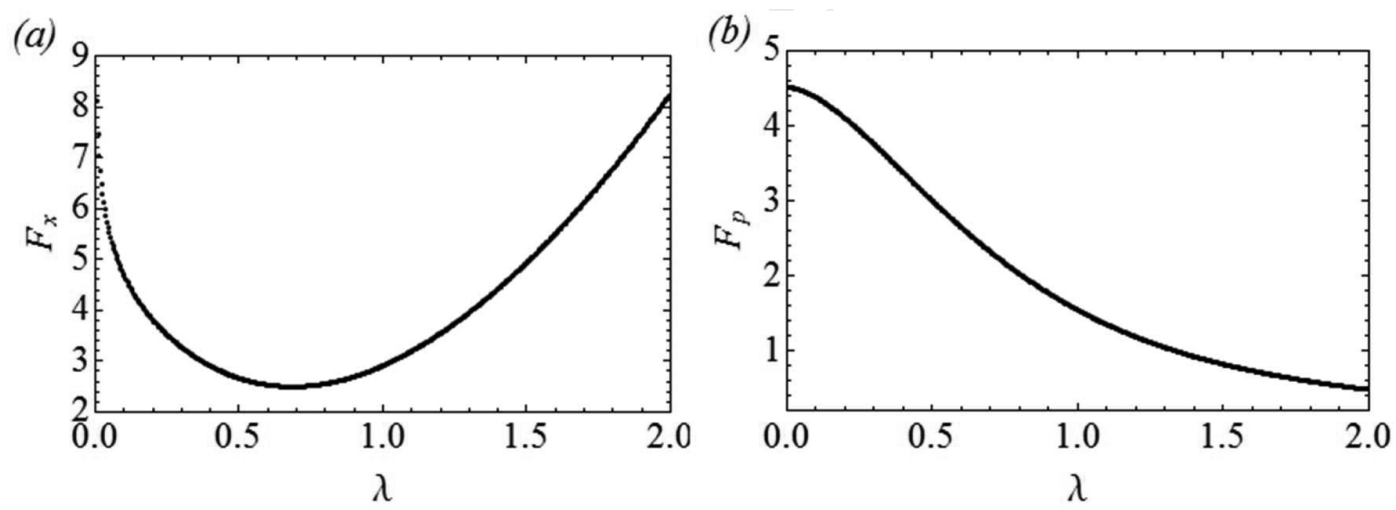

Figura 4: Dependência das informações de Fisher (a) $F_{x}$ e (b) $F_{p}$ com relação ao parâmetro $\lambda$. Nos cálculos consideramos $m_{0}=1$.
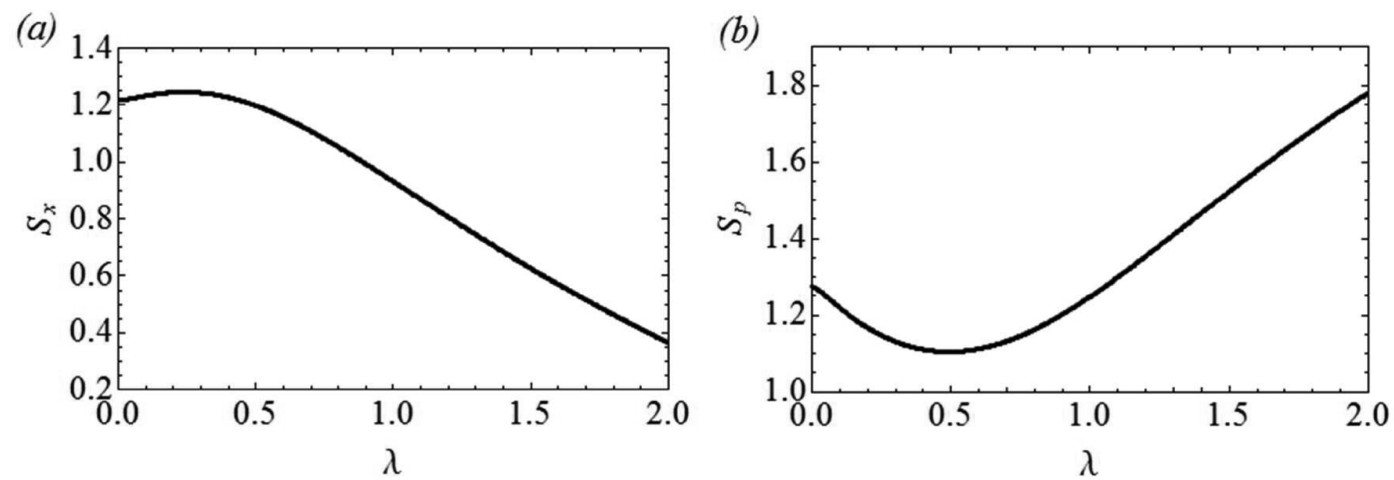

Figura 5: Dependência das entropias de Shannon (a) $S_{x}$, e (b) $S_{p}$ com relação ao parâmetro $\lambda$. Nos cálculos consideramos $m_{0}=1$.

quanto $F_{p}$ diminuem enquanto $\lambda$ aumenta. A partir de $\lambda=0,6, F_{x}$ aumenta e $F_{p}$ diminui.

Consideremos agora o comportamento de $S_{x}$ e $S_{p}$ mostrado nas Figs. 5(a) e 5(b), respectivamente. Observamos que no intervalo $0 \leqslant \lambda \leqslant 0,4, S_{x}$ tem um pequeno aumento, enquanto $S_{p}$ diminui de forma mais acentuada. Já no intervalo $0,4<\lambda \leqslant 2$, observamos que $S_{x}$ diminui, enquanto que $S_{p}$ aumenta.

Para entendermos esses resultados, consideremos, mais uma vez, o comportamento de $\mathcal{P}_{0}(x)$ com relação ao parâmetro $\lambda$ mostrado nas Figs.1(a) e 2 (a). Observamos que, no intervalo $0 \leqslant \lambda \leqslant 1,0$, a dispersão nos valores de posição da partícula tornase menos deformada na origem e sua largura mais estreita. Como $\mathcal{P}_{0}(x)$ torna-se mais localizado com o aumento de $\lambda$, é razoável supor que os comportamentos de $F_{x}, F_{p}, S_{x}$ e $S_{p}$ observados no intervalo $0 \leqslant \lambda \leqslant 0,6$, sejam por causa da deformação que ocorre em $\mathcal{P}_{0}(x)$ para pontos próximos da origem. Para $\lambda>0,6$, os comportamentos de $F_{x}, F_{p}, S_{x}$ e $S_{p}$ correspondem àqueles esperados quando ocorre uma diminuição na dispersão da posição. Em outras pa- lavras, a partícula fica mais localizada, aumentando $F_{x}$ e diminuindo $S_{x}$.

As deformações observadas em $\mathcal{P}_{0}(x)$, por causa da forma de $m(x)$, não são percebidas pela incerteza $\Delta x_{0}$ (veja Fig. 1(a)) porque o integrando $x^{2} P_{0}(x)$ é sempre nulo na origem. Para verificarmos a consistência dessa explicação, vamos analisar o comportamento de $\mathcal{P}_{0}(x)$ em função de $\lambda$ para a distribuição de massa considerada na Ref.[6], ou seja, $m(x)=m_{0}\left[1+(\lambda x)^{2}\right]$, pois nesse sistema as quantidades $F_{x}, F_{p}, S_{x}$ e $S_{p}$ são bem comportadas, no sentido que sempre crescem ou decrescem quando $\lambda$ varia. Nas Figs. 6(a) e 6(b) mostramos o comportamento de $\mathcal{P}_{0}(x)$ para (a) $\lambda=0, \lambda=0,2$ e $\lambda=0,4$ e (b) $\lambda=0,6, \lambda=0,8$ e $\lambda=1$. Observamos que $\mathcal{P}_{0}(x)$ não apresenta deformações, pois independentemente do valor de $\lambda$, a massa deste sistema nunca se anula, seja na origem ou em qualquer outro valor de posição.

Mas, ao contrário de $\Delta x_{0}$, a incerteza $\Delta p_{0}$ percebe as deformações. Da mesma maneira que $\Delta x_{0}$, o 

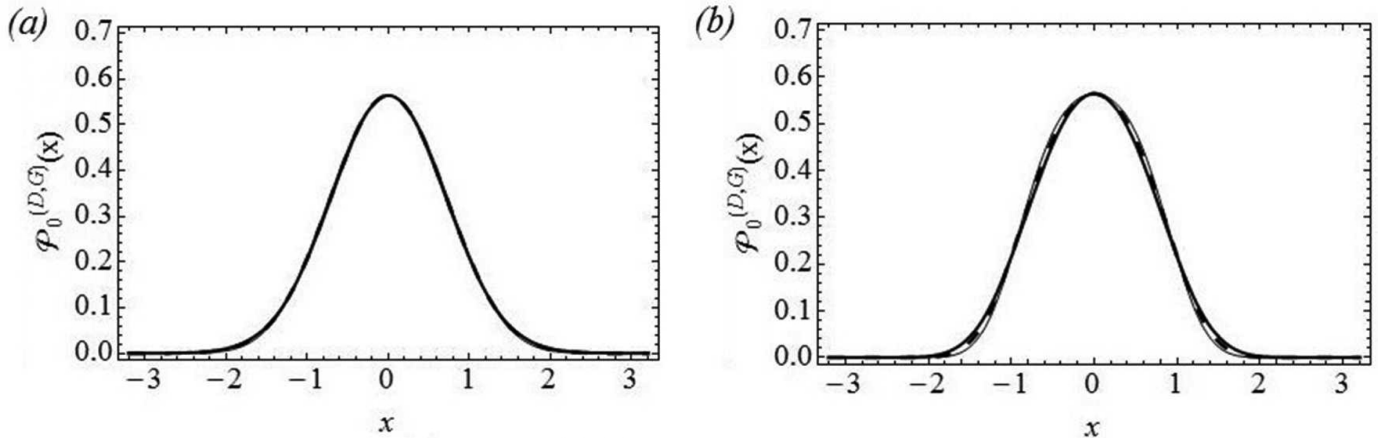

Figura 6: Variação de $\mathcal{P}_{0}(x)$ para o sistema $m(x)=m_{0}\left[1+(\lambda x)^{2}\right]$, para (a) $\lambda=0$ (linha grossa), $\lambda=0.2$ (linha pontilhada) e $\lambda=0.4$ (linha fina) e (b) $\lambda=0.6$ (linha grossa), $\lambda=0.8$ (linha pontilhada) e $\lambda=1$ (linha fina). Nos cálculos consideramos $m_{0}=1$.

desvio padrão $\Delta p_{0}$ depende do integrando $p^{2} \mathcal{P}_{0}(p)$. A diferença está no fato que as deformações de $\mathcal{P}_{0}(p)$ não se encontram em $p=0$, mas sim nas regiões $-3.5 \leqslant p \leqslant-1.5$ e $1.5 \leqslant p \leqslant 3.5$ (veja as Figs. 1(b) e 2(b)). Assim, como nas outras quantidades, atribuímos o comportamento não usual de $\Delta p_{0}$, no intervalo $0 \leqslant \lambda \leqslant 0,6$, a essas deformações. A partir de $\lambda>0,6$ as deformações em $\mathcal{P}_{0}(p)$ desaparecem e o comportamento de $\Delta p_{0}$ passa a concordar com o que é esperado quando $\Delta x_{0}$ diminui.

\section{Conclusões}

Neste trabalho estudamos o oscilador harmônico quântico com massa dependente da posição (OMDP). Utilizamos a álgebra de operadores escada juntamente com o método da TCP para obter a função de onda exata de um OMDP. Este procedimento consiste em decompor a hamiltoniana que descreve o sistema quântico OMDP (Eq. (16)) em termos de dois operadores auto-adjuntos, $A_{a}^{+}$e $A_{a}^{-}$. Utilizando as definições de $A_{a}^{+}$e $A_{a}^{-}$(Eqs. 19(a) e 19(b)) e impondo a álgebra de Heisenberg sobre estes operadores, obtivemos uma expressão geral para o potencial $V_{a}(x)$ (Eq. (25)) em termos da distribuição de massa $m(x)$ e da constante de vínculo $a$. Para que haja concordância entre as expressões quântica (Eq. (25)) e clássica (Eq. (26)) de $V_{a}(x)$, escolhemos $a=-1 / 4$. De posse das expressões de $T(x)$ e $V_{-1 / 4}(x)$, obtivemos a equação diferencial associada a equação de Schrödinger de um OMDP (Eq. (32)), cuja solução pode ser obtida reduzindo-a a equação de autovalores do oscilador harmônico com massa constante através das transformações expressas pelas Eqs. (33) e (34).
Em particular, consideramos o sistema com distribuição de massa dada por $m(x)=m_{0}\left(x^{2}+\lambda^{2}\right)$. Após obtermos a função de onda, determinamos numericamente as informações de Fisher, as entropias de Shannon além das incertezas para o estado fundamental deste sistema. Tanto a entropia de Shannon quanto a informação de Fisher estão sendo investigadas como possíveis substitutas das incertezas que aparecem no princípio de incerteza de Heisenberg.

Observamos que ao aumentarmos $\lambda, \Delta x_{0}$ diminui. Entretanto o comportamento de $\Delta p_{0}, F_{x}, F_{p}, S_{x}$ e $S_{p}$ é um pouco mais complexo. Se $\Delta x_{0}$ sempre diminui, $F_{x}$ e $\Delta p_{0}$ deveriam sempre aumentar e $S_{x}$ diminuir. Atribuímos o comportamento não usual de $\Delta p_{0}, F_{x}$, $F_{p}, S_{x}$ e $S_{p}$ no intervalo $0 \leqslant \lambda \leqslant 0,6$, às deformações que surgem nas densidades de probabilidade $\mathcal{P}_{0}(x)$ e $\mathcal{P}_{0}(p)$ em função da forma de $m(x)$ considerada. Para $\lambda>0,6$, quando as deformações tornam-se menores e desaparecem, os comportamentos de $\Delta p_{0}$, $F_{x}, F_{p}, S_{x}$ e $S_{p}$ correspondem àqueles esperados quando $\Delta x_{0}$ diminui.

Confirmamos esta hipótese, analisando o comportamento de $\mathcal{P}_{0}(x)$ em função de $\lambda$, para o sistema estudado na Ref. [6], onde $m(x)=m_{0}\left[1+(\lambda x)^{2}\right]$. Como mostrado nas Figs. 6(a) e 6(b), $\mathcal{P}_{0}(x)$ não apresenta nenhuma distorção em qualquer valor de posição, e, como consequência os comportamentos de $\Delta p_{0}, F_{x}, F_{p}, S_{x}$ e $S_{p}$ seguem os previstos para quando $\Delta x_{0}$ diminui. Observamos que a igualdade na inequação de Stam (Eq. (43)) é satisfeita para qualquer valor de $\lambda$, enquanto que a igualdade na inequação de Cramer-Rao (Eq. (44)) é satisfeita apenas para $\lambda>1,1$. 


\section{Agradecimentos}

Os autores agradecem ao CNPQ pelo auxílio financeiro.

\section{Referências}

[1] R. Koç, M. Koca and G. Sahinoglu, Eur. Phys. J. B 48, 583 (2005).

[2] G. Bastard, Wave Mechanics Applied to Semiconductor Heterostructures (Les Editions de Physique, Les Ulis, 1988).

[3] D.L. Smith and C. Mailhiot, Rev. of Mod. Phys. 62, 173 (1990).

[4] L.I. Serra and E. Lipparini, Europhys. Lett. 40, 667 (1997).

[5] F.A. Saavedra, J. Boronat, A. Polls and S. Fabrocini, Phys. Rev. B 50, 4282 (1994).

[6] D.X. Macedo and I. Guedes, Physica A 434, 211 (2015).

[7] B. Bagchi, P. Gorain, C. Quesne and R. Roychoudhury, Modern Phys. Lett. A 19, 2765 (2004).

[8] K.C. Yung and J.H. Yee, Phys. Rev. A 50, 104 (1994).

[9] J.P.G. Nascimento e I. Guedes, Revista Brasileira de Ensino de Física 36, 4308 (2014).

[10] O. von Roos, Phys. Rev. B 27, 7547 (1983).

[11] D.J. BenDaniel and C.B. Duke Phys. Rev. B 152, 683 (1966).

[12] T. Gora and F. Williams, Phys. Rev. 177, 1179 (1969).

[13] Q.G. Zhu and H. Kroemer, Phys. Rev. B 27, 3519 (1983).

[14] T. Li and K.J. Kuhn, Phys. Rev. B 27, 3519 (1983).

[15] D.J. Griffiths, Introduction to Quantum Mechanics (Prentice Hall, New Jersey, 2005), 2nd ed.

[16] W. Heisenberg, Z. Phys. 43, 172 (1927).

[17] R.A. Fisher, Proc. Cambridge Philos. Soc. 22, 700 (1925).

[18] C. Shannon and W. Weaver, A Mathematical Theory of Communication (Urbana, 1949).

[19] A.L. Martín, J.C. Angulo and J. Antolín, Physica A 392, 5552 (2013).

[20] A.J. Stam, Inf. Control 2, 101(1959).

[21] A. Dembo, T.M. Cover and J.A. Thomas, IEEE Trans. Inform. Theory 37, 1501 (1991).

[22] S. Dong, Guo-Hua Sun, Shin-Hai Dong and J.P. Draayer, Phys. Lett. A 378, 124 (2014).

[23] I. Bialynicki-Birula and J., Mycielski, Comm. Math. Phys. 44, 129 (1975).

[24] W. Beckner, Ann. of Math. 102, 159 (1975). 